\title{
To Ascertain Factors of Learning Objectives Facilitating Quality Education Factor Analysis and Modelling the Relationship Among the Factors by Regression Model
}

\author{
Sheetal Aditya ${ }^{1 *}$ and Neha Singh ${ }^{2}$ \\ ${ }^{1}$ Department of Management, Shri Jagdishprasad Jhabarmal Tibrewala University, Rajasthan, India \\ ${ }^{2}$ Department of Management, Dr. Rammanohar Lohia Avadh University, Uttar Pradesh, India
}

"Corresponding author: sheetal.adityas@gmail.com

$\begin{array}{lll}\text { Received: 21-12-2020 Revised: 17-02-2021 Accepted: 28-03-2021 } & \text { A }\end{array}$

\begin{abstract}
The relationship between learning objective of online learning acquired by students and learning outcome achieved by them is studied through understanding the association 24 factors in learning benefits and learning satisfaction. Learning objective under the research were assumptions or statements which are expected to be achieved by a student or learner at the end of a course or unit or qualification. They can be consequences a student or learner must know, evaluate and be able to exhibit after completion of a process of learning. Association of factors were studied through Exploratory Factor Analysis with principal component with 173 respondents of students with Kaiser-Meyer-Olkin Measure of Sampling Adequacy value of 0.932 and Bartlett's Test of Sphericity giving 0.00 sig. value. The Cronbach's alpha is 0.995, which indicates a high level of internal consistency in the variable of study to understand online learning among students. After the rotation and extraction of fixed 3 factor component, given by scree plot and Varimax with Kaiser Normalization, F1 factors are derived comprising of 12 factors. These 12 factors were studied through linear regression model assuming these 12 factors as independent variable on dependent variable significant benefit in online learning. The model summary gave the values of $R=$ 0.665 , R Square $=0.443$ and Adjusted R Square $=0.401$ with ANNOVA significant value $=0.00$. With the backward regression applied of range of 0.05 to 0.51 , there were 8 excluded factors.

The regression equation can be predicated as follows:-

$Y$ (Significant benefit in online learning $)=a($ Constant $)+b 1$ (Coefficient of Better Learning retention) $\times 1$ (Better Learning retention) $+b 2$ (Coefficient of Cost effective score) $\times 2$ (Cost effective score) $+b 3$ (Coefficient of Helps to increase interest) $\times 3$ (Helps to increase interest) $+b 4$ (Coefficient of Learning on own's pace) $\times 4$ (Learning on own's pace) $+b 5$ (Coefficient of Mobility and ease of use of online learning tools) $\times 5$ (Mobility and ease of use of online learning tools).
\end{abstract}

Keywords: Online learning benefits, Online learning, Linear Regression Model, Exploratory Factor Analysis, learning objective

The demand of creative and Innovative pedagogical approach in today's pandemic has increased greatly. The teaching and learning process is embracing several innovations like use of technology through blended learning (combination of face-to-face and online teaching and learning). Online learning which has been a component of Open and Distance learning (ODL) institution is now getting weight age among most conventional higher education providers as well as among students. Advancement and creation in Information communication technology as well as in Instructional Design method have given

How to cite this article: Aditya, S. and Singh, N. (2021). To Ascertain Factors of Learning Objectives Facilitating Quality Education Factor Analysis and Modelling the Relationship Among the Factors by Regression Model. Educational Quest: An Int. J. Edu. Appl. Soc. Sci., 12(1): 01-08.

Source of Support: None; Conflict of Interest: None 
result in designing of e-learning platforms that can be used to present or effective deliver learning content with recent and enhanced opportunities for student engagement, interaction and learning (Ituma 2011). (Paetcher 2010) Proposed that such frameworks must have at least five segments for designing an e-learning course including: (1) course design, learning materials and electronic course environment; (2) communication among between stake holder: (3) communication between student peers; (4) Personal learning framework; and (5) course outcomes result. Studies have shown that students' online experience can be academically challenge.ng (Dobbs 2009) (Wyatt 2005).

The programmes offered by any education provider must be evaluated in order to ensure the effectiveness of the programme in achieving its objectives like learning objective, learning satisfaction and learning benefits. During such flow one largest obstacle is about how the stakeholders can successfully adopt the usage of technology and ensuring participants' contribution given the each learner nature and encounters with advancement of technology (Hofmann, 2014). The notion of using innovation and technology along with blended learning, need inclusive with reinforcement of factors of learning objective along with learning satisfaction and learning benefit. The study focuses on understanding the factors achieving learning objective along with learning satisfaction and learning benefits.

Today's modification in Education can become transformative when stakeholder i.e., Teachers and students synthesize and synergise information across subjects and experiences, critically weigh significantly different perspectives, and incorporate various inquiries. Educators and felicitator areable to construct opportunities by applying critical learning spaces, in which students are encouraged to enhance their capacities of creative analysis, imagination, and critical synthesis, creative expression of thoughts, self-awareness, and intentionality.

\section{Objectives of the study}

To study the learning benefits and satisfaction acquired in online learning among undergraduate students facilitating quality education.

\section{Hypothesis of the study:-}

H0: There is no significant learning and satisfaction benefits acquired in online learning among undergraduate students

$\square \mathrm{Ha}$ - There is a significant learning benefits and satisfaction acquired in online learning among undergraduate students

\section{Review of Literature}

\begin{tabular}{|c|c|c|c|}
\hline $\begin{array}{l}\text { S1. } \\
\text { No. }\end{array}$ & $\begin{array}{l}\text { Title of research } \\
\text { article with } \\
\text { author name }\end{array}$ & Summary & Key words \\
\hline 1 & $\begin{array}{l}\text { The impact of } \\
\text { online interaction } \\
\text { on student } \\
\text { learning outcome } \\
\text { Chou (Chou, } \\
\text { Peng, \& Chang, } \\
\text { 2010) }\end{array}$ & $\begin{array}{l}\text { Has defined active interaction } \\
\text { in online learning activities } \\
\text { including the types of } \\
\text { interaction: the learner -self } \\
\text { learner, among different } \\
\text { learner- learner, interaction } \\
\text { between learner - instructor, } \\
\text { exchange among learner } \\
\text {-content, and learner interface. } \\
\text { The results of their study } \\
\text { showed that students with } \\
\text { better results and need less } \\
\text { time learning when interacting } \\
\text { more with the system }\end{array}$ & $\begin{array}{l}\text { Online learning } \\
\text { activities, } \\
\text { learning } \\
\text { analytics, } \\
\text { blended } \\
\text { learning }\end{array}$ \\
\hline 2 & \begin{tabular}{|l|} 
Factors of learner \\
instructor \\
interaction which \\
predict perceived \\
learning outcomes \\
in online learning \\
environment \\
Kang and \\
colleagues (Kang \\
$\&$ Im, 2013)
\end{tabular} & $\begin{array}{l}\text { The interactive activities } \\
\text { between teachers and } \\
\text { students have an great } \\
\text { impact on various learning } \\
\text { outcomes process of students } \\
\text { while implementing } \\
\text { number of learning modes } \\
\text { such as various learning } \\
\text { assistance, \& others social } \\
\text { intimacy, interaction \& other } \\
\text { instructional Q \& A, teachers } \\
\text { presence. }\end{array}$ & $\begin{array}{l}\text { Student } \\
\text { - teacher } \\
\text { interaction, } \\
\text { traditional } \\
\text { teaching, } \\
\text { blended } \\
\text { learning } \\
\text { environment }\end{array}$ \\
\hline 3 & \begin{tabular}{|l|} 
A Study on \\
teachers' \\
perceptions \\
of and their \\
satisfaction with \\
interaction type in \\
blended learning \\
environments by \\
Brian R. Schroder, \\
Andrew E.
\end{tabular} & $\begin{array}{l}\text { Walker and Belland, Kerstin } \\
\text { E. Blended learning is an } \\
\text { approach that combines } \\
\text { technology based learning. } \\
\text { With face-to-face interactions, } \\
\text { Within their article, the } \\
\text { blended learning model } \\
\text { approach. }\end{array}$ & $\begin{array}{l}\text { Blended } \\
\text { learning, } \\
\text { technology } \\
\text { based learning }\end{array}$ \\
\hline 4 & \begin{tabular}{|l|} 
Factors that \\
influence \\
students' decision \\
to drop-out of \\
online courses \\
(Willging \& \\
Johnson, 2009)
\end{tabular} & $\begin{array}{l}\text { Research shows that absence } \\
\text { of learner interaction causes } \\
\text { failure and eventual drop-out } \\
\text { in online courses and the lack } \\
\text { of learner connectedness was } \\
\text { noted as an another internal } \\
\text { factor leads to different } \\
\text { learner drop-out in various } \\
\text { online Program. It was } \\
\text { found that learners may not } \\
\text { continue in e-learning and } \\
\text { blended learning if they fail } \\
\text { to make friends thereby being } \\
\text { disconnected and enhancing } \\
\text { feelings of separation during } \\
\text { the process of blended } \\
\text { learning. }\end{array}$ & $\begin{array}{l}\text { Feelings of } \\
\text { isolation, } \\
\text { blended } \\
\text { learning } \\
\text { experiences, } \\
\text { learner } \\
\text { interaction }\end{array}$ \\
\hline
\end{tabular}




\begin{tabular}{|c|c|c|c|}
\hline 5 & $\begin{array}{l}\text { The Effect of } \\
\text { Using Blended } \\
\text { Learning on the } \\
\text { Achievement } \\
\text { of Students and } \\
\text { Information } \\
\text { Retention of Fifth } \\
\text { Graders in the } \\
\text { Biology Course } \\
\text { Maccoun, Hussein } \\
\text { Salem (2016). }\end{array}$ & $\begin{array}{l}\text { Paper aimed to find the effect } \\
\text { of using the blended learning } \\
\text { in students' achievement and } \\
\text { information preservation } \\
\text { for the fifth graders in the } \\
\text { biology course. The results } \\
\text { showcase the superiority } \\
\text { of the experimental group } \\
\text { to the control group in } \\
\text { the achievement test and } \\
\text { information retention. }\end{array}$ & \begin{tabular}{|l} 
Students' \\
achievement, \\
Information \\
preservation, \\
Information \\
retention
\end{tabular} \\
\hline 6 & $\begin{array}{l}\text { The Effect of } \\
\text { Using Blended } \\
\text { Learning in } \\
\text { Teaching English } \\
\text { Language } \\
\text { on the Direct } \\
\text { and Delayed } \\
\text { Achievement } \\
\text { among the } \\
\text { Sixth Graders. } \\
\text { Al-Rimawi, Firas } \\
\text { Tharwat (2014). }\end{array}$ & $\begin{array}{l}\text { Author aimed to focus the } \\
\text { effect of blended learning } \\
\text { on the direct and delayed } \\
\text { achievement of the sixth } \\
\text { graders in the English } \\
\text { language course and to } \\
\text { achieve the objectives of } \\
\text { the study and the quasi } \\
\text { experimental approach was } \\
\text { adopted. The study also } \\
\text { showed the presence and } \\
\text { achievement of statistically } \\
\text { relevant significant differs } \\
\text { among the means of the } \\
\text { direct \&delayed purpose and } \\
\text { achievement for the group of } \\
\text { the experimental team. }\end{array}$ & $\begin{array}{l}\text { Blended } \\
\text { learning, Direct } \\
\text { and Delayed } \\
\text { achievement. }\end{array}$ \\
\hline 7 & $\begin{array}{l}\text { Critical thinking, } \\
\text { cognitive } \\
\text { presence, and } \\
\text { computer } \\
\text { conferencing } \\
\text { in distance } \\
\text { education. } \\
\text { Garrison, D. R., } \\
\text { Anderson, T. \& } \\
\text { Archer, W. (2009). }\end{array}$ & $\begin{array}{l}\text { The researcher motivating } \\
\text { empirical results related to } \\
\text { various attempt to create an } \\
\text { efficient and most reliable } \\
\text { source of instrument to pass } \\
\text { the features \& quality of } \\
\text { critical outcome and related } \\
\text { thinking in a text- based } \\
\text { educational context. The } \\
\text { authors suggest that cognitive } \\
\text { presence (i.e., critical, practical } \\
\text { inquiry) can be created and } \\
\text { supported in a computer- } \\
\text { conference environment with } \\
\text { appropriate teaching and } \\
\text { social presence }\end{array}$ & $\begin{array}{l}\text { Reliable } \\
\text { instrument, } \\
\text { cognitive } \\
\text { presence }\end{array}$ \\
\hline 8 & $\begin{array}{l}\text { Online education: } \\
\text { Best practices to } \\
\text { promote learning. } \\
\text { Finch, D. \& } \\
\text { Jacobs, K. (2012). }\end{array}$ & $\begin{array}{l}\text { Qualitative method was } \\
\text { used to collect the relevant } \\
\text { responses. This paper } \\
\text { discusses best practices \& } \\
\text { evidence literature related } \\
\text { to online education. Big } \\
\text { Level of quality educational } \\
\text { experiences in human related } \\
\text { factors \&ergonomics (HFE) } \\
\text { are of interest to the global } \\
\text { ergonomics community in } \\
\text { reference to promote the } \\
\text { development of the high } \\
\text { profession, enhance the related } \\
\text { skill set of HFE followers, and } \\
\text { facilitate the translation of } \\
\text { knowledge into practice. }\end{array}$ & $\begin{array}{l}\text { Online } \\
\text { education, } \\
\text { global } \\
\text { ergonomics } \\
\text { community, } \\
\text { human factors } \\
\text { and ergonomics }\end{array}$ \\
\hline
\end{tabular}

\begin{tabular}{|c|c|c|c|}
\hline 9 & $\begin{array}{l}\text { The Impact of } \\
\text { Blended Learning } \\
\text { on Student } \\
\text { Outcomes: is } \\
\text { there room on the } \\
\text { horse for two? } \\
\text { J. Mitchell O'Toole } \\
\text { \& Douglas J. } \\
\text { Absalom }\end{array}$ & $\begin{array}{l}\text { The research exists regarding } \\
\text { the impact of ICT on the } \\
\text { achievement of student } \\
\text { outcomes in specific } \\
\text { undergraduate courses, } \\
\text { particularly how student } \\
\text { utilization of varying modes } \\
\text { within blended provision } \\
\text { relates to their achievement } \\
\text { of course outcomes. The } \\
\text { study shows that ICT access } \\
\text { formats by themselves are of } \\
\text { limited benefit in achieving } \\
\text { course outcomes, The study } \\
\text { also provides some insight } \\
\text { into the complexities of the } \\
\text { blending flow in attempting } \\
\text { to incorporate new advance } \\
\text { technology into recent } \\
\text { teaching scenario, and trying } \\
\text { to identify more practical } \\
\text { path to accept in advancing } \\
\text { the process of technological } \\
\text { teaching. }\end{array}$ & $\begin{array}{l}\text { ICT, Blended } \\
\text { Learning } \\
\text { process, } \\
\text { practical } \\
\text { directions, } \\
\text { technological } \\
\text { teaching. }\end{array}$ \\
\hline 10 & $\begin{array}{l}\text { Online education } \\
\text { and its effective } \\
\text { practice: A } \\
\text { research review. } \\
\text { Sun, A. \& Chen, } \\
\text { X. (2016). }\end{array}$ & $\begin{array}{l}\text { Qualitative content analysis } \\
\text { approach was used in } \\
\text { assessments of online learning } \\
\text { environment. The paper } \\
\text { shows actual recommendation } \\
\text { for those who are thinking } \\
\text { to develop online available } \\
\text { courses so that they can come } \\
\text { across informed decisions in } \\
\text { the implementation. Based } \\
\text { on the authors findings, } \\
\text { the authors portrayed that } \\
\text { effective online learning } \\
\text { instruction is dependent } \\
\text { upon (a) very well-developed } \\
\text { designed course content, } \\
\text { motivated talk between } \\
\text { the instructor and learners, } \\
\text { another fully learned and } \\
\text { fully-supported order and } \\
\text { instructors; (b) making and } \\
\text { creating of a sense of online } \\
\text { learning community; and (c) } \\
\text { great move in development } \\
\text { technology. }\end{array}$ & $\begin{array}{l}\text { Online } \\
\text { education } \\
\text { process, online } \\
\text { teaching } \\
\text { frame work, } \\
\text { online learning } \\
\text { community } \\
\text { and group, } \\
\text { asynchronous } \\
\text { learning, } \\
\text { cognitive } \\
\text { presence and } \\
\text { attainment, } \\
\text { social } \\
\text { presence and } \\
\text { achievement, } \\
\text { teaching } \\
\text { presence, } \\
\text { online higher } \\
\text { education } \\
\text { institution. }\end{array}$ \\
\hline
\end{tabular}

\section{Research Methodology}

Primary Data Sources: Questionnaire devised for students among Management, Information Technology and Commerce Students under graduation level from different colleges.

SPSS Software used for testing Linear Regression Model

\section{Descriptive Statistics}

$\square$ The mean, mode, median and range

Variance and standard deviation

\section{Frequencies}

Cross tabs 


\section{Reliability analysis using Cronbach's Alpha}

Secondary Data Sources: Research papers, Journals, Reports, webliography links

Sampling: Purposive Sampling Method

Sample: 174 respondents

\section{Data Interpretation and Data Analysis}

1. Understanding devices used for online learning by students across Commerce, Management, Mass Media and IT/CS

\begin{tabular}{|c|c|c|c|c|c|c|c|}
\hline \multicolumn{8}{|c|}{ Devices used for online learning } \\
\hline \multicolumn{8}{|l|}{ Count } \\
\hline & & \multicolumn{5}{|c|}{ Devices used for online learning } & \multirow[t]{2}{*}{ Total } \\
\hline & & & 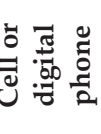 & 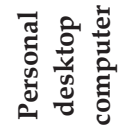 & 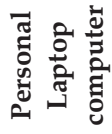 & 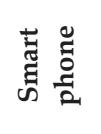 & \\
\hline \multicolumn{3}{|c|}{ Commerce } & 2 & 1 & 4 & 44 & 51 \\
\hline \multirow{3}{*}{$\begin{array}{l}\text { Stream } \\
\text { of study }\end{array}$} & IT/CS & & 0 & 1 & 0 & 0 & 1 \\
\hline & dy Manageme & & 0 & 2 & 5 & 37 & 44 \\
\hline & Mass Med & & 5 & 3 & 11 & 59 & 78 \\
\hline \multicolumn{2}{|l|}{ Total } & & 7 & 7 & 20 & 140 & 174 \\
\hline \multicolumn{8}{|c|}{ Devices Used for Online learning } \\
\hline & & \multicolumn{2}{|c|}{ Frequency } & Percent & $\begin{array}{l}\text { Valid } \\
\text { Percent }\end{array}$ & \multicolumn{2}{|c|}{$\begin{array}{l}\text { Cumulative } \\
\text { t Percent }\end{array}$} \\
\hline \multirow{5}{*}{ Valid } & $\begin{array}{l}\text { Cell or digital } \\
\text { phone }\end{array}$ & 7 & & 4.0 & 4.0 & 4.0 & \\
\hline & $\begin{array}{l}\text { Personal } \\
\text { desktop } \\
\text { computer }\end{array}$ & 7 & & 4.0 & 4.0 & 8.0 & \\
\hline & $\begin{array}{l}\text { Personal } \\
\text { Laptop } \\
\text { computer }\end{array}$ & 20 & & 11.5 & 11.5 & 19.5 & \\
\hline & Smart phone & 140 & & 80.5 & 80.5 & 100.0 & \\
\hline & Total & 174 & & 100.0 & 100.0 & & \\
\hline
\end{tabular}

Interpretation: $80.5 \%$ of students use smart phones as device for online learning

2. To understand Skill level of students under various streams of study

\begin{tabular}{|c|c|c|c|c|c|c|}
\hline \multicolumn{7}{|c|}{ Skill Level of students under Email usage } \\
\hline \multicolumn{7}{|l|}{ Count } \\
\hline & & \multicolumn{4}{|c|}{ Email } & \multirow[b]{2}{*}{ Total } \\
\hline & & $\begin{array}{l}\text { Do not } \\
\text { use }\end{array}$ & Skilled & Unskilled & $\begin{array}{l}\text { Very } \\
\text { Skilled }\end{array}$ & \\
\hline \multirow{4}{*}{$\begin{array}{l}\text { Stream } \\
\text { of study }\end{array}$} & Commerce & 10 & 29 & 7 & 5 & 51 \\
\hline & IT/CS & 0 & 0 & 0 & 1 & 1 \\
\hline & Management & 5 & 29 & 3 & 7 & 44 \\
\hline & Mass Media & 3 & 60 & 5 & 10 & 78 \\
\hline Total & & 18 & 118 & 15 & 23 & 174 \\
\hline
\end{tabular}

\begin{tabular}{|c|c|c|c|c|c|c|}
\hline \multicolumn{7}{|c|}{ Skill Level of students under Web surfing } \\
\hline \multicolumn{7}{|l|}{ Count } \\
\hline & & \multicolumn{4}{|c|}{ Web surfing } & \multirow[b]{2}{*}{ Total } \\
\hline & & $\begin{array}{l}\text { Do not } \\
\text { use }\end{array}$ & Skilled & $\begin{array}{l}\text { Un- } \\
\text { skilled }\end{array}$ & $\begin{array}{l}\text { Very } \\
\text { Skilled }\end{array}$ & \\
\hline \multirow{4}{*}{$\begin{array}{l}\text { Stream of } \\
\text { study }\end{array}$} & Commerce & 5 & 31 & 10 & 5 & 51 \\
\hline & IT/CS & 0 & 0 & 0 & 1 & 1 \\
\hline & Management & 7 & 20 & 6 & 11 & 44 \\
\hline & Mass Media & 4 & 48 & 12 & 14 & 78 \\
\hline Total & & 16 & 99 & 28 & 31 & 174 \\
\hline \multicolumn{7}{|c|}{$\begin{array}{l}\text { Skill Level of students under Word Processing, Power point } \\
\text { Presentation, Excel }\end{array}$} \\
\hline \multicolumn{7}{|l|}{ Count } \\
\hline & & \multicolumn{4}{|c|}{$\begin{array}{l}\text { Word Processing, Power point } \\
\text { Presentation, Excel }\end{array}$} & \multirow{2}{*}{ - Total } \\
\hline & & $\begin{array}{l}\text { Do not } \\
\text { use }\end{array}$ & Skilled & $\begin{array}{l}\text { Un- } \\
\text { skilled }\end{array}$ & $\begin{array}{l}\text { Very } \\
\text { Skilled }\end{array}$ & \\
\hline \multirow{4}{*}{$\begin{array}{l}\text { Stream of } \\
\text { study }\end{array}$} & Commerce & 14 & 26 & 8 & 3 & 51 \\
\hline & IT/CS & 0 & 1 & 0 & 0 & 1 \\
\hline & Management & 5 & 26 & 3 & 10 & 44 \\
\hline & Mass Media & 6 & 47 & 18 & 7 & 78 \\
\hline Total & & 25 & 100 & 29 & 20 & 174 \\
\hline
\end{tabular}

\section{Interpretation}

The students of skilled with usage of Email, Web surfing for information and Word Processing, Power point Presentation, Excel and it implies students rely on this tools for learning.

\section{Reliability of Data using Cronbach's Alpha}

\begin{tabular}{lll}
\hline \multicolumn{3}{c}{ Reliability Statistics } \\
\hline Cronbach's Alpha & $\begin{array}{l}\text { Cronbach's } \\
\text { Alpha Based on } \\
\text { Standardized Items }\end{array}$ & N of Items \\
\hline .891 & .892 & 6 \\
\hline
\end{tabular}

Item Statistics

\begin{tabular}{llll}
\hline & Mean & Std. Deviation & N \\
\hline Learning on own's pace & 3.95 & .961 & 174 \\
Helps to increase interest & 3.48 & 1.068 & 174 \\
$\begin{array}{l}\text { Mobility and ease of use of } \\
\text { online learning tools }\end{array}$ & .971 & 174 \\
$\begin{array}{l}\text { Cost effective } \\
\begin{array}{l}\text { Better Learning retention } \\
\text { Significant benefit in }\end{array}\end{array}$ & 3.69 & .971 & 174 \\
online learning & 4.10 & .795 & 174 \\
\hline
\end{tabular}

\begin{tabular}{llll}
\hline \multicolumn{3}{c}{ Scale Statistics } \\
\hline Mean & Variance & Std. Deviation & N of Items \\
\hline 22.82 & 21.238 & 4.608 & 6 \\
\hline
\end{tabular}




\section{Interpretation}

Cronbach's alpha is the common measure of internal consistency ("reliability") as Cronbach's alpha is 0.891 , which indicates a high level of internal consistency in the variable of study to understand online learning benefits among students.

\section{Linear Regression Analysis}

\section{Variables:-}

Dependent Variable: Significant benefit in online learning

\section{Independent Variables}

(a) Learning on own's pace

(b) Helps to increase interest

(c) Mobility and ease of use of online learning tools

(d) Cost effective

(e) Better Learning retention

\section{Output under SPSS}

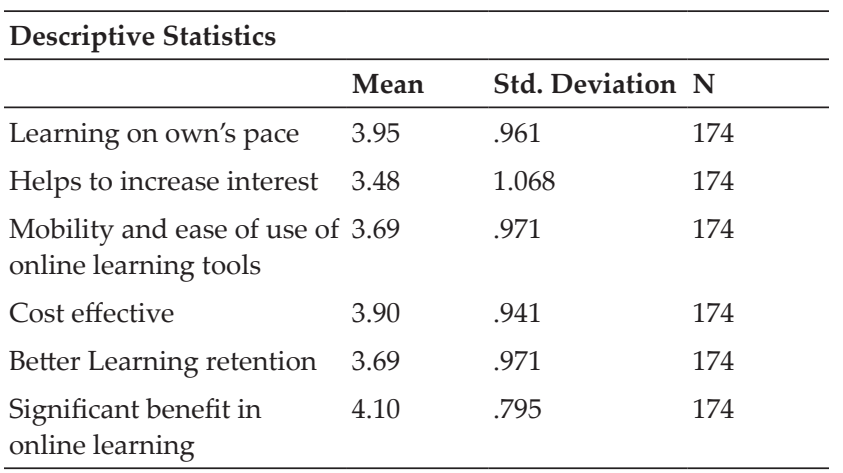

\section{Correlations}

\begin{tabular}{|c|c|c|c|c|c|c|c|}
\hline & & 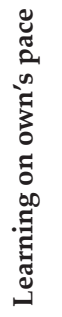 & 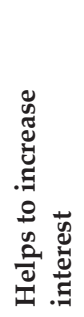 & 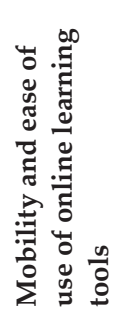 & 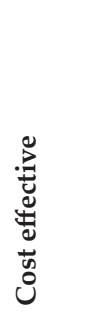 & 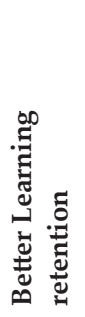 & 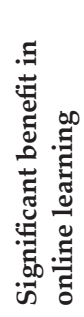 \\
\hline \multirow{3}{*}{$\begin{array}{l}\text { Learning } \\
\text { on own's } \\
\text { pace }\end{array}$} & $\begin{array}{l}\text { Pearson } \\
\text { Correlation }\end{array}$ & 1 & $.568^{* *}$ & $.666^{* *}$ & $.513^{* *}$ & $.641^{* *}$ & $.846^{* *}$ \\
\hline & $\begin{array}{l}\text { Sig. } \\
\text { (2-tailed) }\end{array}$ & & .000 & .000 & .000 & .000 & .000 \\
\hline & $\mathrm{N}$ & 174 & 174 & 174 & 174 & 174 & 174 \\
\hline
\end{tabular}

Helps to increase interest

Mobility
and ease
of use of
online
learning
tools

Cost effective

Pearson $\quad .568^{* *} 1 \quad .685^{* *} \quad .490^{* *} .596^{* *} .448^{* *}$

Correlation

$\begin{array}{llllll}\text { Sig. } & .000 & .000 & .000 & .000 & .000\end{array}$

(2-tailed)

$\begin{array}{lllllll}\mathrm{N} & 174 & 174 & 174 & 174 & 174 & 174\end{array}$

Pearson

$.666^{* *} .685^{* *} 1 \quad .567^{* *} .681^{* *} .541^{* *}$

Correlation

$\begin{array}{llllll}\text { Sig. } & .000 & .000 & .000 & .000 & .000\end{array}$

(2-tailed)

$\begin{array}{lllllll}\mathrm{N} & 174 & 174 & 174 & 174 & 174 & 174\end{array}$

Pearson $\quad .513^{* *} .490^{* *} \quad .567^{* *} \quad 1 \quad 1542^{* *} .438^{* *}$

Correlation

$$
\begin{array}{lllll}
.000 & .000 \quad .000 \quad 000 & .000
\end{array}
$$

Sig.

(2-tailed)

\begin{tabular}{llllllll} 
& $\mathrm{N}$ & 174 & 174 & 174 & 174 & 174 & 174 \\
& $\begin{array}{l}\text { Pearson } \\
\text { Correlation }\end{array}$ & $.641^{* *} .596^{* *}$ & $.681^{* *}$ & $.542^{* *} 1$ & $.474^{* *}$ \\
$\begin{array}{l}\text { Better } \\
\text { Learning }\end{array}$ & $\begin{array}{l}\text { Sig. } \\
\text { retention }\end{array}$ & .000 & .000 & .000 & .000 & & .000 \\
& $(2$-tailed) & & & & & & \\
& $\mathrm{N}$ & 174 & 174 & 174 & 174 & 174 & 174 \\
& $\begin{array}{l}\text { Pearson } \\
\text { Significant }\end{array}$ & $.846^{* *} .448^{* *}$ & $.541^{* *}$ & $.438^{* *} .474^{* *}$ & 1 \\
$\begin{array}{l}\text { Correlation } \\
\text { in online } \\
\text { learning }\end{array}$ & $\begin{array}{l}\text { Sig. } \\
\text { (2-tailed) }\end{array}$ & .000 & .000 & .000 & .000 & .000 & \\
& $\mathrm{~N}$ & 174 & 174 & 174 & 174 & 174 & 174 \\
\hline
\end{tabular}

*. Correlation is significant at the 0.01 level (2-tailed).

\begin{tabular}{|c|c|c|c|}
\hline \multicolumn{4}{|c|}{ Variables Entered/Removed ${ }^{a}$} \\
\hline Model & Variables Entered & Variables Removec & d Method \\
\hline 1 & $\begin{array}{l}\text { Better Learning } \\
\text { retention, Cost } \\
\text { effective, Helps to } \\
\text { increase interest, } \\
\text { Learning on own's } \\
\text { pace, Mobility } \\
\text { and ease of use } \\
\text { of online learning } \\
\text { tools }^{\text {b }}\end{array}$ & . & Enter \\
\hline \multicolumn{4}{|c|}{$\begin{array}{l}\text { a. Dependent Variable: Significant benefit in online learning } \\
\text { b. All requested variables entered. }\end{array}$} \\
\hline \multicolumn{4}{|c|}{ Model Summary } \\
\hline Model & R Square & $\begin{array}{l}\text { Adjusted R } \\
\text { Square }\end{array}$ & $\begin{array}{l}\text { Std. Error of } \\
\text { the Estimate }\end{array}$ \\
\hline 1 & $.852^{\mathrm{a}}$ & .717 & .423 \\
\hline
\end{tabular}




\begin{tabular}{|c|c|c|c|c|c|c|}
\hline \multicolumn{7}{|c|}{ ANOVA $^{a}$} \\
\hline \multicolumn{2}{|c|}{ Model } & $\begin{array}{l}\text { Sum of } \\
\text { Squares }\end{array}$ & df & $\begin{array}{l}\text { Mean } \\
\text { Square }\end{array}$ & $\mathbf{F}$ & Sig. \\
\hline \multirow{3}{*}{1} & Regression & 79.323 & 5 & 15.865 & 88.796 & $.000^{\mathrm{b}}$ \\
\hline & Residual & 30.016 & 168 & .179 & & \\
\hline & Total & 109.339 & 173 & & & \\
\hline
\end{tabular}

a. Dependent Variable: Significant benefit in online learning

b. Predictors: (Constant), Better Learning retention, Cost effective, Helps to increase interest, Learning on own's pace, Mobility and ease of use of online learning tools

\section{Backward Regression Output}

\begin{tabular}{|c|c|c|c|}
\hline \multicolumn{4}{|c|}{ Variables Entered/Removed ${ }^{a}$} \\
\hline Model & Variables Entered & $\begin{array}{l}\text { Variables } \\
\text { Removed }\end{array}$ & Method \\
\hline 1 & $\begin{array}{l}\text { Better Learning } \\
\text { retention, Cost effective, } \\
\text { Helps to increase } \\
\text { interest, Learning on } \\
\text { own's pace, Mobility } \\
\text { and ease of use of online } \\
\text { learning tools }\end{array}$ & . & Enter \\
\hline 2 & . & $\begin{array}{l}\text { Mobility and } \\
\text { ease of use of } \\
\text { online learning } \\
\text { tools }\end{array}$ & $\begin{array}{l}\text { Backward } \\
\text { (criterion: } \\
\text { Probability of } \\
\text { F-to-remove } \\
>=.100) .\end{array}$ \\
\hline 3 & . & $\begin{array}{l}\text { Helps to } \\
\text { increase interest }\end{array}$ & $\begin{array}{l}\text { Backward } \\
\text { (criterion: } \\
\text { Probability of } \\
\text { F-to-remove } \\
>=.100) .\end{array}$ \\
\hline 4 & . & Cost effective & $\begin{array}{l}\text { Backward } \\
\text { (criterion: } \\
\text { Probability of } \\
\text { F-to-remove } \\
>=.100) .\end{array}$ \\
\hline
\end{tabular}

a. Dependent Variable: Significant benefit in online learning

b. All requested variables entered.

\begin{tabular}{lllll}
\hline \multicolumn{5}{c}{ Model Summary } \\
\hline Model & $\mathbf{R}$ & R Square & $\begin{array}{l}\text { Adjusted R } \\
\text { Square }\end{array}$ & $\begin{array}{l}\text { Std. Error of } \\
\text { the Estimate }\end{array}$ \\
\hline 1 & $.852^{\mathrm{a}}$ & .725 & .717 & .423 \\
2 & $.852^{\mathrm{b}}$ & .725 & .719 & .421 \\
3 & $.852^{\mathrm{c}}$ & .725 & .720 & .420 \\
\hline 4 & $.851^{\mathrm{d}}$ & .724 & .721 & .420 \\
\hline
\end{tabular}

a. Predictors: (Constant), Better Learning retention, Cost effective, Helps to increase interest, Learning on own's pace, Mobility and ease of use of online learning tools

b. Predictors: (Constant), Better Learning retention, Cost effective, Helps to increase interest, Learning on own's pace

c. Predictors: (Constant), Better Learning retention, Cost effective, Learning on own's pace

d. Predictors: (Constant), Better Learning retention, Learning on own's pace

\begin{tabular}{|c|c|c|c|c|c|c|}
\hline & & & NOV & & & \\
\hline & lel & Sum of & df & Mean & $\mathbf{F}$ & Sig. \\
\hline & Regression & 79.323 & 5 & 15.865 & 88.796 & $.000^{\mathrm{b}}$ \\
\hline 1 & Residual & 30.016 & 168 & .179 & & \\
\hline & Total & 109.339 & 173 & & & \\
\hline & Regression & 79.316 & 4 & 19.829 & 111.618 & $.000^{c}$ \\
\hline 2 & Residual & 30.023 & 169 & .178 & & \\
\hline & Total & 109.339 & 173 & & & \\
\hline & Regression & 79.294 & 3 & 26.431 & 149.553 & $.000^{\mathrm{d}}$ \\
\hline 3 & Residual & 30.045 & 170 & .177 & & \\
\hline & Total & 109.339 & 173 & & & \\
\hline & Regression & 79.157 & 2 & 39.579 & 224.240 & $.000^{\mathrm{e}}$ \\
\hline 4 & Residual & 30.182 & 171 & .177 & & \\
\hline & Total & 109.339 & 173 & & & \\
\hline
\end{tabular}

a. Dependent Variable: Significant benefit in online learning

b. Predictors: (Constant), Better Learning retention, Cost effective, Helps to increase interest, Learning on own's pace, Mobility and ease of use of online learning tools

c. Predictors: (Constant), Better Learning retention, Cost effective, Helps to increase interest, Learning on own's pace

d. Predictors: (Constant), Better Learning retention, Cost effective, Learning on own's pace

e. Predictors: (Constant), Better Learning retention, Learning on own's pace

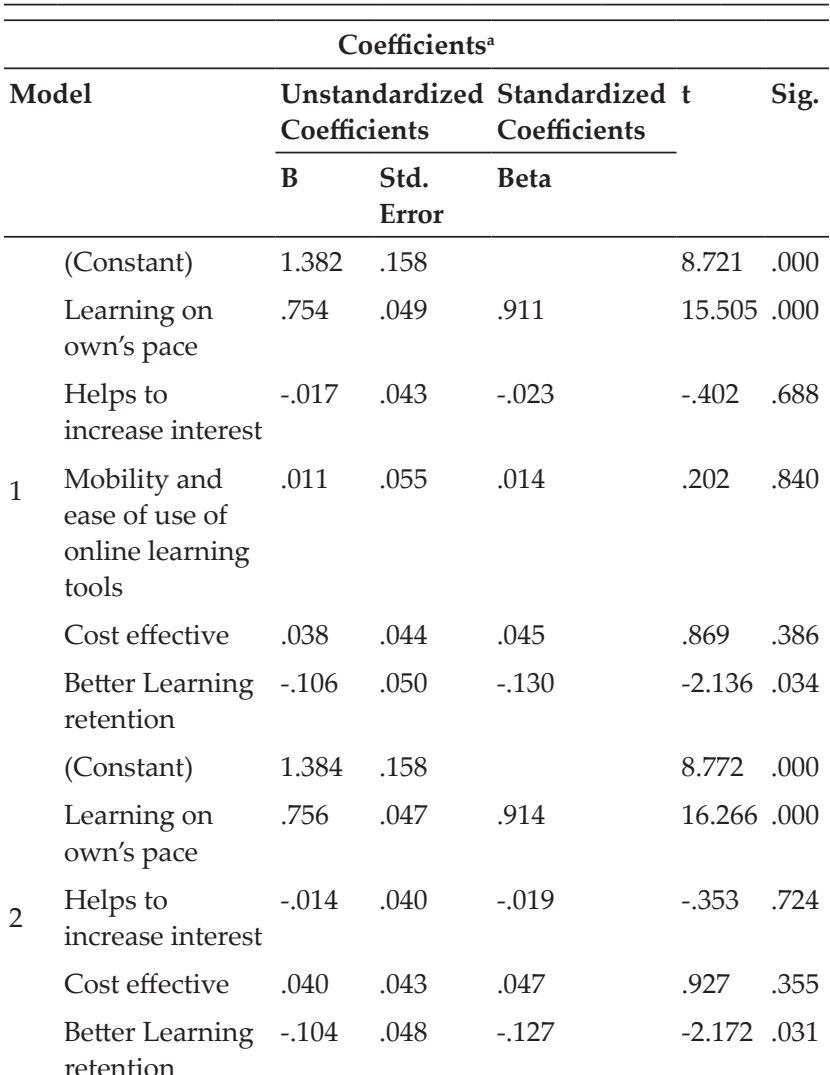




\begin{tabular}{|c|c|c|c|c|c|c|}
\hline & (Constant) & 1.381 & .157 & & 8.788 & .000 \\
\hline & $\begin{array}{l}\text { Learning on } \\
\text { own's pace }\end{array}$ & .752 & .045 & .909 & 16.769 & .000 \\
\hline & Cost effective & .037 & .042 & .044 & .879 & .381 \\
\hline & $\begin{array}{l}\text { Better Learning } \\
\text { retention }\end{array}$ & -.109 & .045 & -.133 & -2.399 & .018 \\
\hline & (Constant) & 1.437 & .144 & & 10.004 & .000 \\
\hline 4 & $\begin{array}{l}\text { Learning on } \\
\text { own's pace }\end{array}$ & .762 & .043 & .921 & 17.593 & .000 \\
\hline & $\begin{array}{l}\text { Better Learning } \\
\text { retention }\end{array}$ & -.096 & .043 & -.117 & -2.236 & .027 \\
\hline
\end{tabular}

\section{Interpretation}

(a) H0: There is no significant learning and satisfaction benefits acquired in online learning among undergraduate students using factors such as Learning on own's pace, Helps to increase interest, Mobility and ease of use of online learning tools, Cost effective and Better Learning retention, as the score in ANNOVA is less than 0.05 we reject the null hypothesis.

(b) Initially in the model all the variables were entered with the Dependent variable i.e. Significant benefit in online learning i.e. Learning on own's pace, Helps to increase interest, Mobility and ease of use of online learning tools, Cost effective and Better Learning retention.

(c) With the backward regression applied of range of 0.05 to 0.51 , the first excluded variable was Mobility and ease of use of online learning tools then in the second excluded variable along with Helps to increase interest and third excluded variable was Cost effective.

(d) Correlation tables gives the values.

\section{Correlation}

\begin{tabular}{|c|c|c|c|c|c|c|c|}
\hline & & 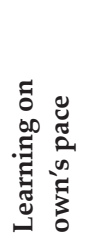 & 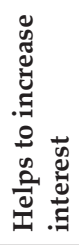 & 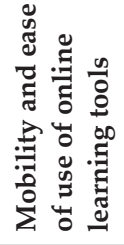 & 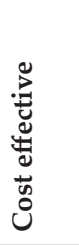 & 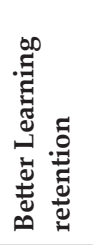 & 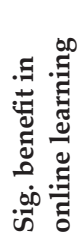 \\
\hline \multirow{3}{*}{$\begin{array}{l}\text { Sig. } \\
\text { benifit } \\
\text { in online } \\
\text { learning }\end{array}$} & $\begin{array}{l}\text { Pearson } \\
\text { Correlation }\end{array}$ & $0846^{* *}$ & $.448^{*}$ & $.541^{* *}$ & $.438^{*}$ & $.474^{* *}$ & 1 \\
\hline & $\begin{array}{l}\text { Sig. } \\
\text { (2-tailed }\end{array}$ & .000 & .000 & .000 & .000 & .000 & \\
\hline & $\mathrm{N}$ & 174 & 174 & 174 & 174 & 174 & 174 \\
\hline
\end{tabular}

**. Correlation is significant at the level (2-tailed).
So, it can be derived that impact on Significant benefit in online learning by Learning on own's pace is 0.846 , Helps to increase interest 0.448 , Mobility and ease of use of online learning tools is 0.541, Cost effective is 0.438 and Better Learning retention 0.474.

(e) As the sigma value in Annova table is less than 0.05 we can reject the null hypothesis and test it further

(f) The adjusted $R$ value

\begin{tabular}{lllll}
\hline \multicolumn{3}{l}{ Model Summary } \\
\hline Model & $\mathbf{R}$ & R Square & $\begin{array}{l}\text { Adjusted R } \\
\text { Square }\end{array}$ & $\begin{array}{l}\text { Std. Error of } \\
\text { the Estimate }\end{array}$ \\
\hline 1 & $.852^{\mathrm{a}}$ & .725 & .717 & .423 \\
2 & $.852^{\mathrm{b}}$ & .725 & .719 & .421 \\
3 & $.852^{\mathrm{c}}$ & .725 & .720 & .420 \\
4 & $.851^{\mathrm{d}}$ & .724 & .721 & .420 \\
\hline a. Predictors: (Constant), Better Learning retention, Cost effective, Helps \\
to increase interest, Learning on own's pace, Mobility and ease of use of \\
online learning tools \\
b. Predictors: (Constant), Better Learning retention, Cost effective, Helps \\
to increase interest, Learning on own's pace \\
c. Predictors: (Constant), Better Learning retention, Cost effective, Learning \\
on own's pace \\
d. Predictors: (Constant), Better Learning retention, Learning on own's pace \\
\hline
\end{tabular}

The adjusted $R$ value is close to 0.8 that is 0.721 so linear regression model can be used for predication with the least square method.

$\mathrm{Y}$ (Significant benefit in online learning) $=\mathrm{a}$ (Constant) + b1 (Coefficient of Better Learning retention) $x 1$ (Better Learning retention) $+\mathrm{b} 2$ (Coefficient of Cost effective score ) x2 (Cost effective score) $+b 3$ (Coefficient of Helps to increase interest) x3 (Helps to increase interest) $+\mathrm{b} 4$ (Coefficient of Learning on own's pace) $x 4$ (Learning on own's pace) + b5 (Coefficient of Mobility and ease of use of online learning tools) x5 (Mobility and ease of use of online learning tools)

But in Backward method Mobility and ease of use of online learning tools, Helps to increase interest and Cost effective was removed so the equation becomes:-

$\mathrm{Y}$ (Significant benefit in online learning) $=\mathrm{a}$ (Constant) + b1 Coefficient of Better Learning retention) x1 (Better Learning retention) $+\mathrm{b} 4$ (Coefficient of Learning on own's pace) x4 (Learning on own's pace) 
The regression equation can be predicated as follows:-

\begin{tabular}{|c|c|c|c|c|c|c|}
\hline \multirow[t]{3}{*}{4} & (Constant) & 1.437 & .144 & & 10.004 & .000 \\
\hline & $\begin{array}{l}\text { Learning on } \\
\text { own's pace }\end{array}$ & .762 & .043 & .921 & 17.593 & .000 \\
\hline & $\begin{array}{l}\text { Better Learning } \\
\text { retention }\end{array}$ & -.096 & .043 & -.117 & -2.236 & .027 \\
\hline
\end{tabular}

a. Dependent variable: Significant benefit in online learning.

$\mathrm{Y}$ (Significant benefit in online learning $)=\mathrm{a}$ (Constant) + b1 Coefficient of Better Learning retention) $\mathrm{x} 1$ (Better Learning retention) $+\mathrm{b} 4$ (Coefficient of Learning on own's pace) $x 4$ (Learning on own's pace)

$\mathrm{Y}$ (Significant benefit in online learning) $=\mathrm{a}$ (Constant) + b1Coefficient of Better Learning retention) $x 1$ (Better Learning retention) $+\mathrm{b} 4$ (Coefficient of Learning on own's pace) x4 (Learning on own's pace)

$\mathrm{Y}$ (Significant benefit in online learning $)=1.437$ $+0.762 \times 1$ (Better Learning retention) - $0.96 \times 4$ (Learning on own's pace)

\section{Recommendations and Suggestions}

Through the statistical analysis it is evident that online learning has gained immense importance in education system by its acceptance by students and teachers as well.

There are various multifaceted online learning mediums available according to learning pattern, teaching pattern, students' ability etc.

$\square$ Online learning mediums which would radically change the traditional pedagogy for prospective future technological change happening

Students would benefit immensely with the integration of online learning as it would help them learn and grasp effectively

Challenges of traditional pedagogy can be minimized or reduced

Under the various available online learning mediums for students according to Factor Analysis explains that an online learning medium becomes relevant for students if factors of Quality learning, learning retention, Better Communication and Understanding of concepts are focused on.

\section{REFERENCES}

Al-Rimawi, Firas Tharwat. 2014. The Effect of Using BlendedLearning in Teaching English Language on the Direct and Delayed Achievement among the Sixth Graders, Unpublished Master Thesis, Faculty of Educational Sciences, Middle East University, Jordan.

Chou, C., Peng, H. and Chang, C.Y. 2010. The technical framework of interactive functions for course-management systems: Students' perceptions, uses, and evaluations. Computers and Education, 55(3): 1004-1017.

Dobbs, R.R., Waid, C.A. and del Carmen, A. 2009. "Students' perceptions of online courses: the effect of online." Quarterly Review of Distance Education, 10(1): 9-26,89,91.

Finch, D. and Jacobs, K. 2012. Online education: Best practices to promote learning. Proceedings of the Human Factors and Ergonomics 56 $6^{\text {th }}$ Annual Meeting.

Garrison, D.R., Anderson, T. and Archer, W. 2009. Critical thinking, cognitive presence, and computer conferencing in distance education. American Journal of Distance Education, 15(1): 7-23.

Ituma, A. 2011. "An evaluation of student's perceptions and engagement with e-learning components in a campus." 12(1): 57-68.

J. Mitchell O'Toole \& Douglas J. Absalom. 2003. The Impact of Blended Learning on Student Outcomes: is there room on the horse for two?, Journal of Educational Media, 28: 2-3, 179-190.

Maccoun, Hussein Salem. 2016. The Effect of Using Blended Learning on the Achievement of Students and Information Retention of Fifth Graders in the Biology Course, Faculty of Education Journal, 22(95): 209-240.

Paetcher, M., Maier, B. and Macher, D. 2010. "Students" expectations of, and experiences in e-learning: Their." 54(1): 21-40.

Sun, A. and Chen, X. 2016. Online education and its effective practice: A research review. Journal of Information Technology Education: Research, 15: 157-190

Wyatt, G. 2005. "Satisfaction, academic rigor and interaction: perceptions of online instruction. Education." 125(3). 